\title{
Carnets
}

Revue électronique d'études françaises de l'APEF

Deuxième série - 13 | 2018

Corps, rythmes et voix : en/jeux littéraires et artistiques

\section{Une poétique et une didactique des relations de Voix}

Enjeux et perspectives pour l'enseignement et la recherche

\section{Serge Martin}

\section{(2) OpenEdition \\ Journals}

Édition électronique

URL : http://journals.openedition.org/carnets/2651

DOI : 10.4000/carnets. 2651

ISSN : 1646-7698

Éditeur

APEF

\section{Référence électronique}

Serge Martin, «Une poétique et une didactique des relations de Voix», Carnets [En ligne], Deuxième série - 13 | 2018, mis en ligne le 31 mai 2018, consulté le 19 avril 2019. URL : http:// journals.openedition.org/carnets/2651 ; DOI : 10.4000/carnets.2651

Ce document a été généré automatiquement le 19 avril 2019

\section{(c) () () (9)}

Carnets est mis à disposition selon les termes de la licence Creative Commons - Atribution - Pas d'utilisation commerciale 4.0 International. 


\title{
Une poétique et une didactique des relations de Voix
}

Enjeux et perspectives pour l'enseignement et la recherche

\author{
Serge Martin
}

\section{Avant-dire avec Robert Desnos}

1 Un poème de Robert Desnos, extrait de Contrées dont l'achevé d'imprimer du 31 mai 1944, soit quelques mois après l'arrestation de son auteur, le 22 février de la même année, a pour titre « La voix ${ }^{1} »$.

La voix

Une voix, une voix qui vient de si loin

Qu'elle ne fait plus tinter les oreilles,

Une voix, comme un tambour, voilée

Parvient pourtant, distinctement, jusqu'à nous.

Bien qu'elle semble sortir d'un tombeau

Elle ne parle que d'été et de printemps,

Elle emplit le corps de joie,

Elle allume aux lèvres le sourire.

Je l'écoute. Ce n'est qu'une voix humaine

Qui traverse les fracas de la vie et des batailles,

L'écroulement du tonnerre et le murmure des bavardages.

Et vous? Ne l'entendez-vous pas?

Elle dit « La peine sera de courte durée »

Elle dit « La belle saison est proche».

Ne l'entendez-vous pas? (Desnos, 1999 : 1171)

2 De voix à vous, le poème engage une écoute de l'infime comme passage, que l'adresse forte et itérative finale construit jusqu'à ouvrir l'infini de l'écho que le poème signalait dès l'incipit : «la voix» c'est « une voix, une voix». Desnos n'a pas tout dit mais il a tout 
engagé de ce que j'aimerais partager avec vous : il n'y a pas de voix sans réénonciation, pas de voix sans racontage, sans l'histoire d'une voix qui n'en finit pas de faire écho jusque dans l'expérience plurielle, personnelle ou collective. Et «ce n'est qu'une voix humaine » qui «vient de si loin» et qui «parvient pourtant, distinctement, jusqu'à nous ». Mais Desnos nous aura prévenus avec ce consonantisme du négatif, ce bégaiement de l'écoute qui doit sans cesse refaire le long chemin traversé de voix à vous, de peine à proche par-delà les apparences et les fracas... Cette épopée de l'infime, voilà ce que chaque relation de voix engage en poétique et en didactique, d'un même mouvement. Voilà résumés les enjeux et les perspectives d'un tel engagement pour l'enseignement et la recherche à mille lieues des grandes orgues des sciences de la littérature et de l'enseignement. Il nous faut alors prendre le temps d'écouter dans l'infini reprise où les doublons prosodiques font l'écho, la rime si l'on préfère : « Une voix, comme un tambour, voilée / parvient pourtant, distinctement, jusqu'à nous. »

3 Je vous propose un parcours en trois moments: 1) la voix fait relation contre les habitudes discontinuistes, séparatrices et demande de penser l'oralité comme test du faire littérature, c'est-à-dire du faire voix ; 2) faut-il pour autant lancer un tournant vocal après d'autres? je ne le pense pas, apprendre à écouter que la voix fait la vie - ce serait toute la poétique -, obligerait à entendre que les vies demandent de faire voix ; 3 ) alors les critiques comme les didactiques arrimées à un tel travail poétique, éthique et politique, n'auraient qu'à accompagner au plus près les points de voix pour en augmenter les résonances : de quoi « emplir le corps de joie » et « allumer aux lèvres le sourire » pour et par des racontages, des passages de voix et d'expériences, sans fin.

\section{Du discontinu au continu : la voix fait relation}

4 Les arts du langage depuis toujours font attention à la voix (Pierra, 2006) mais généralement cette attention est arrimée à une conception dualiste de la voix. En effet, soit elle se porte vers l'appareil phonatoire-respiratoire du corps et ainsi la vocalité se voit réduite à une acoustique quand ce n'est pas à des « techniques ${ }^{2}$ ", soit elle engage une métaphore, si ce n'est une transcendance, qui assigne la voix à une incarnation et au mieux à une expression d'un au-delà ou d'un en deçà de la vocalité. On aurait donc depuis des lustres, d'un côté, une rhétorique vocale répondant certes aux cultures de la voix qu'on ne peut nier mais qui réduit la vocalité à un rapport normatif modélisant ou à un rapport expressif mesurable en regard d'un collectivisme vocal ; et, d'un autre côté, une ontologie vocale qui prendrait en charge la force trans-subjectivante des passages de voix entre vox dei et vox populi, c'est-à-dire entre deux métaphysiques dévocalisantes parce que anti-démocratiques, anti-pluralistes. Cette schize est exactement celle que vivent chaque enseignant, chaque élève, pris dans l'étau dualiste du discontinu vocal pratique et théorique avec toutes les conséquences qui s'en suivent: effets de marginalisation de certaines expériences vocales quand elles ne sont pas réduites à du bruit, à un inaudible épistémologique; effets de modélisation chorale où ne s'entend que la voix de son maître et au mieux sa ventriloquie... L'expression qui résumerait le mieux ce dualisme constitutif des conceptions traditionnelles est celle de "techniques et expressions» qui est largement développée dans les formations d'enseignants où la voix est présentée comme un instrument. On peut d'ailleurs observer que ces moments de formation sont pris en charge par les professeurs de musique ${ }^{3}$, parfois même par des professeurs d'EPS, plus rarement par des professeurs de français investis dans les activités théâtrales - autant de 
spécialisations qui technicisent ce qui est de tout le langage et le tout du langage. Presque toutes les disciplines qui se sont intéressé à la voix ont répété un tel dualisme. En critique littéraire on réitère la dichotomie de l'oral et de l'écrit en réduisant l'oralité soit à des cultures périphériques (de la littérature enfantine à l'africaine!), soit au dialogisme bakhtinien qui fait de l'imitation du parlé le nec plus ultra de l'oralité en littérature, que ce soit du côté du roman comme de la poésie appelée "sonore » où la " visuelle » n'aurait donc plus de voix, sans parler de la silencieuse, etc. Il a fallu attendre le maître ouvrage de Henri Meschonnic, Critique du rythme (1982), pour qu'une orientation décisive soit donnée à une poétique de la voix adossée à une anthropologie historique du langage. On a $\mathrm{pu}$ alors passer d'une compréhension sociologique réduisant l'oralité à un mode d'émission, d'exécution et de transmission, à une poétique de l'oralité qui travaille sa théorie du langage par l'attention à l'organisation subjective et culturelle du discours, avec le primat du rythme et de la prosodie, une sémantique propre et donc une écoute de ce que j'appelle la voix-relation. Parce que cette orientation tient à une proposition apparemment des plus simple : «La voix est relation" (op. cit., 294). Elle se reformule ailleurs autrement: "l'énonciation d'un texte qui dure implique une réénonciation infinie » (Meschonnic, 1993 : 114). Bref, la voix fait relation et la relation fait voix : enjeu poétique bien évidemment mais surtout éthique et politique.

5 Je prends un seul exemple que vous connaissez tous, le Cahier d'un retour au pays natal d'Aimé Césaire. Vous savez que ce Cahier commence par cette lancée : «Au bout du petit matin... », lancée reprise plus d'une vingtaine de fois. Et si « c'est au cri que l'on reconnaitt l'homme ", comme écrivait Césaire dans tropiques, en 1941, il ne faut pas attendre quelques vrais cris onomatopéiques comme ceux de la page 30, "voum rooh oh", pour engager la question centrale que lance le Cahier : « Mais qui tourne ma voix? qui écorche ma voix? » Alors que généralement on couvre la force de cette voix par « la question de la négritude ${ }^{4}$ » puisque le Cahier serait le « lieu de l'insurrection de la négritude » et « aussi le lieu de son incarnation » pour y déceler « la dimension militante » de Césaire, laquelle ne se « dissocie pourtant pas de la parole poétique ». C'est ce pourtant qui montre combien le commentaire entretient le discontinu plutôt que de poursuivre une énonciation puissante que les deux questions de Césaire maintiennent fortement dans le continu d'un sujet agissant la vocalité par deux verbes décisifs : « tourner » et « écorcher », c'est-à-dire rimer, danser, infiniment résonner d'une part et d'autre part, dans ce mouvement même de la tourne complètement renversante que l'anaphore initiale lançait, « Au bout du petit matin ", rendre compte d'une terrible souffrance : voix écorchée qui danse, voix dansante d'un écorché vif, voix vive d'une danse écorchante... Aube rimbaldienne ( «J'ai embrassé l'aube d'été »), « et j'ai senti un peu son immense corps ", avec ce couplage consonantique (B/P ; T/T) qui écorche la bouche : oui, le fruit est mûr dans et par cette voix, c'est bien de peau qu'il s'agit et pas de peau si la voix ne tourne pas avec un maximum de corpslangage. Alors non il n'y a pas dans Le Cahier une "parole politique qui s'exprime ", pas plus « la ferveur de la célébration », mais comme l'écrivait Breton dès 1943, " la parole d'Aimé Césaire, belle comme l'oxygène naissant ». Naissance d'une aube vocale, vocalité des commencements, le poème de Césaire engage un sujet qu'on ne peut que découvrir, accompagner dans des recommencements : "Je te suis » écrit in fine Césaire posant non une ontologie à la Descartes mais une anthropologie dans et par le langage à la Benveniste, la relation, rapport et parcours. 


\section{Une voix qui fait la vie: des vies qui font la voix}

6 De quoi avons-nous besoin: d'un tournant vocal après d'autres tournants, subjectif, éthique, relationnel, ou d'une voix qui fait la vie et de vies qui font la voix?

Quel serait l'enjeu d'une poétique de la voix (Rosier-Catach, $2008: 277-284$ ) en regard de ce qu'on a pu appeler, à la suite du tournant subjectif et éthique des sciences humaines et sociales qui a suivi le structuralisme, le tournant relationnel que l'ouvrage de Edouard Glissant, Poétique de la Relation (1990) a pu exemplifier malgré la réification que la majuscule du titre indique. Un tel enjeu commence par une critique des notions qui font la constellation d'une conceptualisation de la voix: sujet, corps, rythme et bien évidemment relation, langage, société. Penser la voix et la relation ensemble, au plus près des pratiques en arts du langage, c'est d'abord penser le corps et la société hors des dualismes et des essentialismes pour augmenter l'attention aux passages possibles des trans-subjectivations vocales ou, autrement dit, des passages de voix qui engagent des passages de sujet. Qu'un devenir voix soit possible dans la relation langagière confirmerait ce qu'un corps fait au langage et ce qu'un discours fait à un corps quand il y a voix et donc poème. C'est toute une gestualité vocale qui viendrait augmenter la relation, en dehors de toute instrumentalisation, qu'elle relève d'un holisme troupier si ce n'est guerrier ou qu'elle soit confinée dans un individualisme même génial. Maria de Jesus Cabral a proposé combien « pour le paradigme de la médecine narrative et de la relation de soins, cela suppose de rester dans l'univers de la parole et du récit moins comme agencement de faits que comme dynamique de corps-langage (Cabral et Almeida, 2017 : 59) " Une telle gestualité, non programmable et encore moins réductible à quelque typologie, demanderait une critique toujours à vif de ses historicités : pas de poétique de la voix qui ne se rejoue chaque fois entièrement à chaque relation de voix. C'est alors qu'on ne peut plus séparer théorie et pratique, poétique et didactique, et encore moins les œuvres des vies. Il n'y a alors qu'à développer chez les élèves comme chez les professeurs l'écoute de ces gestes vocaux, de ce que j'aime appeler des points de voix qui sont comme autant de points de vue engagés par les mouvements de la parole dans le discours.

Un seul exemple avec Ghérasim Luca. Il s'agirait donc de repenser avec des étudiants une expérience décisive dans le champ des expériences dites poétiques, celle de Ghérasim Luca (1913-1993), et de tenter d'en évaluer la portée heuristique pour la poésie aujourd'hui comme vivre poème, extraterritorialité donc et non pouvoir sur les discours et les places. Cette expérience demanderait certainement de considérer un complexe de circonstances : surréalisme roumain et français puis amitiés restreintes et publicisations spectaculaires; identité roumaine puis revendication apatride ; expériences en écritures et en cubomanies; publications typographiées avec le plus grand soin et récitals millimétrés le livre en main... mais également humour et tragique des voix en jeu; théâtralité et scripturalité des poèmes adressés ; éros et thanatos en renversement démonologique... Une telle considération ne viserait pas à mesurer voire maîtriser cette expérience qui ne cesse de travailler bien des expériences en cours, mais à accompagner les performances du poème-Luca comme "essayer dire " défaisant toutes les versions (moderniste ou post-moderniste) de "la performance " pour augmenter l'attention aux gestes et à leurs phrasés ou rythmes de la parole, aux relations et à leurs inventions dialectiques et anachroniques. Il s'agirait en fin de compte de refuser toute axiomatique qui verserait trop vite les performances du poème de Luca dans un style, un procédé, une 
école ou tout autre historicité positive. Ses performances demanderaient au contraire un éventail de temporalisations performatives qui essaient dire (et non de dire) et donc essaient vivre poème (et non de vivre en voire $d u$ poème). De là à en tirer quelques leçons (ce qu'il faut lire) performatives ? Oui, avec Samuel Beckett, « Dire encore » (Cap au pire) ! Mais on se contente d'aller vers un texte qui n'a été publié qu'à l'occasion d'un enregistrement à partir de l'émission télévisuelle réalisée par Raoul Sangla en 1987 (Luca, $2008)^{5}$, en signalant d'ailleurs qu'il s'agit d'un texte que Luca récitait, le livre en main mais le bras le long du corps, et donc ne lisait pas, alors même que tous les autres textes de ses récitals étaient lus livre en main.

Le tangage de ma langue

Des paroles douces

et dès le départ celées :

la conque du silence frôle celle

des récifs...

d'où ce récit

Happé par l'aimant du non-sens

je parle à peu près ceci

pour dire précisément cela

Je suis hélas !

donc on me pense

(L'aveugle vise l'aigle

et tire sur un sourd)

C'est ainsi que je vis

ce que je vois

et que ma voix

se voue au moi qui s'éteint

Comme le «doux » dans le doute

suis-je le «son » de mes songes?

A cette orgie de mots

et d'ascètes à l'écoute

mon Démon sonore agit

sur un monde qui se nie

se noie et se noue

au fond de ma gorge

Sorcier par ondes rythmes

hordes...

Pour le rite de la mort des mots

j'écris mes cris

mes rires pires que fous : faux

et mon éthique phonétique

je la jette comme un sort

sur le langage

En deçà de ceci

et au delà de cela

Hors hors de moi

Car être ailleurs 
tiraille l'heure d'abord

et le mètre ensuite

leur arrêt est ici

mur du son

où l'on fusille un héros

infini

dont la houle cachée

jette un tissu de mots

- un infime drap de mort -

sur le nu d'une muette

couché comme un huit

dans les bras du zéro.

9 Avec ce texte et sa lecture, se réalisent au maximum la définition - ce qu'est «ma langue » - et la valeur - "le tangage ", au sens très précis de ce que fait cette lectureécriture comme force spécifique dans le langage ${ }^{6}$. Le terme "tangage ", qui à une lettre près touche au langage et n'est pas sans évoquer pour tout un chacun le beau titre de Michel Leiris $(1985)^{7}$, est d'emploi courant depuis 1643 pour désigner le mouvement d'un navire qui se balance par un mouvement alternatif et longitudinal (par opposition au roulis) : étymologiquement, Guiraud rapproche le verbe tanguer de l'ancien français tangonner, "piquer de l'aiguillon » qui serait lié au latin tangere, « toucher » et " piquer » au figuré (avec le dérivé tangente), le navire qui tangue « piquant du nez » - informations prises au Dictionnaire historique de la langue française d'Alain Rey). Cette rapide évocation dictionnairique est bel et bien justifiée, et par la « conque du silence » qui « frôle celle des récifs » au début du texte, et par le syntagme « houle cachée » qui intervient à la fin du texte, mais plus certainement par le très ancien mouvement de balancement de tout lecteur dans n'importe quelle yeshivah et, bien au-delà, dans nombres de cultures récitatives - je pense spécialement à celle de l'école primaire où les enseignants s'échinent souvent à empêcher le balancement des élèves récitants... Par ailleurs, il est certainement un écho précis au Salut de Mallarmé et à l'occurrence du terme dans ses derniers vers: Une ivresse belle m'engage Sans craindre même son tangage De porter debout ce salut / Solitude, récif, étoile À n'importe ce qui valut Le blanc souci de notre toile.

Aussi, la valeur qui fait la définition et la définition qui fait la valeur de cette écriturelecture, de ce "ma langue ", c'est-à-dire de ce dire, c'est un tangage qui n'est pas seulement métaphorique ou citationnel, car ce serait en rester à une vision esthétisante ou historicisante de cette écriture-lecture maintenant une dualité fond-forme, quand c'est un mouvement de la parole entièrement "tangage ", au double sens du mouvement d'aller et retour qui « pique de l'aiguillon », qui stimule comme quand on pique les bœufs avec un bâton ferré, et comme "l'aiguillon de la chair» au sens des tentations dans "l'écriture sainte " comme on dit... mais plus certainement comme ce qui incite à un maximum de corps dans le langage. C'est-à-dire un maximum d'« éthique phonétique ", ou de continu forme-sujet ( ma langue ») comme sémantique sérielle, rythmique et prosodique, d'une activité-langage toujours en tangage. Inutile de lister une totalité de procédés quand c'est une force-relation qui organise un tangage de «ceci» à «cela ", d'« à peu près » à " précisément ", de «en deça » à " au delà "... et puis infiniment de vivre à voir, de vocal à vocation, du doute aux rêves, de la négation à la noyade pour que ça ne cesse de se nouer « au fond de ma gorge » dans « hors hors de moi ». Où l'expression n'est pas, comme on dit habituellement dans le confort des pensées du signe, la sortie de soi mais la recherche d'un « hors hors de moi », c'est-à-dire d'un « être ailleurs » comme invention d'un sujet du poème, sujet-relation que seul le poème en écriture-lecture 
invente comme sujet du tangage et donc du langage: «Je suis hélas! / donc on me pense ». Ce « on », ce « Démon sonore » qui « agit / sur un monde qui se nie / se noie et se noue / au fond de ma gorge ", est un "Sorcier par ondes rythmes / hordes... ", où continue la signifiance de l'impersonnel «on» mais également de son activité fondamentale: le «hors» de tout y compris du «hors de moi » même si la colère, l'agitation semblent se poursuivre dans « hordes ». Ce nomadisme du sens ou plutôt du non-sens dont l'aimant happe - jusqu'à un phrasé onomatopéique, est bien ce saisissement par un tangage du récit et donc plutôt du récitatif, de la récitation à tenir, qui paronomase tout ce qui s'en suit pour tout engager au régime d'une résonance générale, rime par tous les bouts, « houle cachée ».

Certes, ce récitatif relève d'un « rite de la mort des mots » et Dominique Carlat a eu raison d'invoquer un "imaginaire respiratoire" de la suffocation chez Luca "qui intériorise l'angoisse de la mort pour la métamorphoser en pulsation du sens (1997 : 256) ». Et Carlat d'évoquer cette conversion de l'angoisse en "rires pires que fous: faux » qu'on lit ici et ailleurs, par exemple dans le Quart d'heure de culture métaphysique qui s'achève par «Expirer en inspirant / Inspirer en expirant». Mais le mouvement qu'on peut dire fondamental du tangage vocal de Luca relève moins « d'une progression régressive (ibid. : 257) », comme le propose Carlat, que d'une force qui va. Alors, l'oralité comme maximum de corps dans le langage associe le zéro et l'infini (certainement une évocation du roman d'Arthur Koestler paru en 1940 et traduit en français en 1945 puisqu'est évoqué un mur et un fusillé voire un tirailleur), un linceul («infime drap de mort») et un nu, un « tissu de mots » et une «muette »; bref le «mur du son » et le «nu d'une muette» où s'entend certainement Le Chant de la carpe dans lequel sont rassemblés le «Quart d'heure de culture métaphysique ", "La paupière philosophale ", "Le verbe", "Passionnément " et " $\mathrm{A}$ gorge dénouée » (Luca: 1986). Au centre de cet ensemble, la théâtralité du "verbe » s'achève sur ce nom de personnage ou plutôt cet appel - comme quand on appelle quelqu'un: "Glissez-glissez-à-votre-tour» (p. 83). En effet, le tangage serait non l'incarnation d'un corps et donc pas du tout la représentation d'une subjectivité même corporelle mais bien la corporalité d'une trans-énonciation pour une utopie d'un tangage infini qui « jette un sort sur le langage », l'ensorcelle « par ondes rythmes / hordes ».

\section{Avec des essais de voix : augmenter les points d'écoute pour multiplier les points de voix}

12 La didactique des littératures ne peut se penser hors d'une poétique dont je viens de donner les linéaments : elle consiste alors à accompagner au plus près de l'expérience des œuvres ce que j'appellerais des réénonciations plurielles. Je me contente ici de quelques pistes critiques pour penser la lecture des textes littéraires comme racontage afin d'engager l'écoute de ce « rapport demeuré rapport » que serait toute œuvre en lectureécriture-diction. Comme le racontage est un passage de voix, c'est au geste vocal que doit se mesurer la force du passage et donc à une activité relationnelle, dans et par le langage (jusque dans ses silences et ses emportements, ses retenues et ses volubilités), qui ouvre à l'approfondissement des historicités de chaque participant au rapport littéraire, au racontage. Ce geste vocal comme passage de voix se constitue comme une transsubjectivation pleine de corps puisqu'un geste c'est un corps qui trouve vie et, mieux, c'est un corps qui fait passer la vie, transforme la vie, tient la vie au plus vif. Par conséquent, aucune topique souvent rapportée à une nomenclature d'actes contrôlables 
ne peut concevoir une gestualité autrement qu'à tenir le geste comme le continu du corps et du langage, du poème et de sa diction, du texte et de sa transmission, de l'écriture et de la lecture. C'est pourquoi, la pensée didactique est d'abord la pensée d'un geste continué ou d'un geste toujours en cours, et rien ne doit venir arrêter ce passage de geste même les meilleures intentions pédagogiques qui souvent contribuer à organiser des prérequis ou autres outils qui empêchent les passages, le continu du racontage, la réénonciation continuée d'une œuvre. Je les appelle des essais de voix.

Alors l'enseignement et la recherche, au plus près des expériences qui co-construisent des points de voix autant que des points de vue, peuvent espérer des mutations décisives pour qu'une poétique des relations de voix constitue le levier d'une politique, d'une éthique et d'une didactique qui augmentent les voix libres dans et par des associations et des individuations ouvertes à l'inconnu vocal de toute rencontre, à ses résonances qui feraient de chacun de ses protagonistes l'écho puisant et pluriel d'un peuple de voix. Aussi, Mallarmé avait-il raison de suggérer combien l'art littéraire était « ce sujet où tout se rattache", à condition qu'on ne le réduise pas à un art séparé des premiers mouvements de la vie d'autant qu'avec Walter Benjamin (2000: 121) on sait combien le racontage comme passage de paroles est passage de voix et donc d'expérience. En fin de compte, les essais de voix sont autant de reprises de voix, au sens de Kierkegaard, des ressouvenirs en avant qui font venir la relation d'inconnu, tout l'inconnu des rapports, des couleurs, des corps, des paroles, du langage. Qui font venir un vivre en voix, une relation dans et par la voix, les voix dans la voix.
Envoi
Il me faudrait toutefois tout reprendre avec un poème.
Je ne sais si entre le sourire du vert été
et ta verte différence il existe une différence
ne sais si je rime par enchantement ou par travaillante
peine. Ne sais si je rime par enchantement ou raison
et ne sais si tu le sais que je rime entièrement
pour toi. Trop de soleil a imprégné la mer dans sa
prison tranquille, où la fleuraille de la mer ne
veut pas mettre main aux bâtiments qui ont sombré.
L'aube lointaine se meut par grisailles. Je ne sais
si entre les pâles rochers je rencontrais le regard,
je ne sais si entre les monotones cris rencontrais
ton regard, je ne sais pas si entre la montagne
et la mer, existe aussi un fleuve. Je ne sais si
entre la côte et le désert se retrouve un fleuve accosté,
je ne sais si à travers la brume tu t'accostes. Je ne
sais si tu tombes ou tu trembles, tu ne sais si je pleure
ou désespère. Désespérer, désespérer, désespérer, tout
ça c'est fabriquer. Tu ne sais pas si je pleure
ou désespère, tu ne sais si je ris ou désespère. Je
ne sais si entre les pâles rochers ton sourire (Rosselli, 2014).

14 Ce poème d'Amelia Rosselli ne cesse de travailler sa résonance par ses variations, ses variations par sa résonance. Il ne cesse également d'engager un non-savoir qui relève d'un emportement dans l'hésitation comme augmentation de la résonance en je-tu au point de "désespérer, désespérer, désespérer » jusqu'à ce qu'apparaisse "ton sourire ", " entre les pâles rochers ». La résonance comme activité d'un infini de la relation tient effectivement de cette tension terrible où «tu t'accostes" peut-être «à travers la brume ». Il y a en effet une obstination de la résonance à faire voix-relation. C'est 
pourquoi, je l'ai appelé en poème ta résonance ${ }^{8}$ car il n'y aurait de résonance vocale que dans et par la relation même impossible - le tu comme appel !

\section{BIBLIOGRAPHIE}

BENJAMIN, Walter (2000). CEuvres III. Paris : Gallimard.

CABRAL, Maria de Jesus (2017). « Dire, de corps en corps. La médecine (narrative) et la question du discours », in Maria de Jesus Cabral et José Domingues de Almeida (éd.) Santé et bien-être à l'épreuve de la littérature. Limoges : Lambert-Luca.

CARLAT, Dominique (1997). «Le tangage de la voix chez Ghérasim Luca », in Voix et création au XX siècle. Paris : Champion pp. 251-261.

DESNOS, Robert CEuvres (1999), Marie-Claire Dumas (éd.). Paris : Gallimard, « Quarto ».

GLISSANT, Edouard (1990). Poétique de la Relation. Paris : Gallimard.

LEIRIS, Michel (1986). Langage tangage ou ce que les mots me disent. Paris : Gallimard.

LUCA, Ghérasim (2008). « Le tangage de ma langue », texte édité dans la pochette du DVD Comment s'en sortir sans sortir, Paris/Genève : José Corti/Héros-Limite [reprise du récital télévisé réalisé par Raoul Sangla. Unité de programme Thierry Garrel, Coproduction CDN Production / La Sept / FR3 Océaniques, diffusé le 20 février 1989 sur la Sept-FR3].

LUCA, Ghérasim (1986). Le Chant de la carpe. Paris : Corti.

MARMONNIER, Evelyne (1994-1995). L'École des Lettres, n5, p. 9 et ss.

MARTIN, Serge (2015). «Ghérasim Luca filmé par Raoul Sangla : la théâtralité du poème.à la télévision ", in Brigitte Denker-Berkoff et alii (dir.). Poésie en scène. éd. Mulhouse : Orizons, coll. «Universités/Comparaisons », pp. 67-80.

MEsCHONNIC, Henri (2000). "La force dans le langage », in Jean-Louis Chiss et Gérard Dessons (dir.). La force du langage Rythme, discours, traduction autour de l'œuvre d'Henri Meschonnic. Paris : Honoré Champion, pp. 9-19.

MESCHONNIC, Henri (1982). Critique du rythme. Lagrasse : Verdier.

MESCHONNIC, Henri (1993). Modernité modernité. Paris : Gallimard.

PIERRA, Gisèle (2006). Le corps, la voix, la texte. Arts du langage en langue étrangère. Paris :

L'Harmattan.

RITMAN, Serge (2017). Ta Résonance, ma retenue. Saint-Benoît-du-Sault : Tarabuste.

ROSIER-CATACH, Irène (2008). "Arts du langage et théologie au Moyen Âge », Annuaire de l'École pratique des hautes études (EPHE), Section des sciences religieuses, nº 115, pp. 277-284.

ROSSELLI, Amelia (2014). La Libellule. Trad. Marie Fabre. Paris : Ypsilon. 


\section{NOTES}

1. Ce poème est le dix-neuvième du recueil Contrées qui en compte vingt-cinq. On peut observer une fiche pédagogique concernant ce poème sur le site de l'académie de Versailles (URL :http:// www.clg-st-exupery-andresy.ac-versailles.fr/IMG/pdf/hda_desnos_la_voix.pdf, consulté le 15 octobre 2017). On observera des différences importantes dans la présentation du poème qui doit faire référence à une autre édition que l'édition "Quarto », mais le plus important concerne l'instrumentalisation du poème et surtout sa dévocalisation : d'abord sous l'angle de l'écart en regard de la norme (« ce poème a pris quelques libertés par rapport aux règles de versification traditionnelles ») puis sous l'angle d'une destinée communicationnelle puisque la voix est réduite à un médium ("Quel message le poète délivre-t-il à travers cette voix?»). Aussi la réduction du « je » au poète et $\mathrm{du}$ « vous » aux « résistants » (voire aux « Français ») périme-t-elle le poème, du moins en réduit l'historicité à un historisme dévocalisant.

2. Voir la fiche "Musique et arts du langage : la voix et ses techniques" pour une classe de cinquième sur le site de l'académie de Lyon: http://www2.ac-lyon.fr/etab/colleges/col-01/ vailland/IMG/pdf/5eme_-_seq_2_-_Doc_de_ref_-

_Musique_et_arts_du_langage_Giroux_2012-2013_.pdf

3. Un exemple parmi de très nombreux autres. Une séquence «Voix, texte et musique au $\mathrm{XX}^{\mathrm{e}}$ siècle ", par Mme Laetitia Antonin, professeure d'éducation musicale au collège Politzer de la

Courneuve (93): http://musique.ac-creteil.fr/IMG/pdf/

Sequence_3oArts_du_langage_techniques_et_expressions.pdf

4. Voir, par exemple, Evelyne Marmonnier, dans L'École des Lettres n 5, 1994-1995, p. 9 et suivantes (les citations sont p. 14 puis p. 15).

5. Texte édité dans la pochette du DVD Comment s'en sortir sans sortir, Paris/Genève, José Corti/ Héros-Limite, 2008 [reprise du récital télévisé réalisé par Raoul Sangla. Unité de programme Thierry Garrel, Coproduction CDN Production / La Sept / FR3 Océaniques, diffusé le 20 février 1989 sur la Sept-FR3]. Voir mon étude : «Ghérasim Luca filmé par Raoul Sangla : la théâtralité du poème à la télévision » dans Brigitte Denker-Berkoff et alii (dir.), Poésie en scène, éd. Orizons, coll. «Universités/Comparaisons », 2015, p. 67-80.

6. Sur la notion de force dans le langage, je renvoie à Henri Meschonnic (2000). «La force dans le langage » dans J.-L. Chiss et G. Dessons (dir.). La force du langage Rythme, discours, traduction autour de l'œuvre d'Henri Meschonnic.

7. Leiris y parle de «vocaliser (son) écriture, la rendre pareille à une voix et donc la 'faire vocale'« (p. 125)!

8. Voir Ritman, Serge (2017). Ta Résonance, ma retenue. Saint-Benoît-du-Sault : Tarabuste.

\section{RÉSUMÉS}

Cet article propose un parcours en trois moments. Tout d'abord, il s'agit de poser l'hypothèse que la voix fait relation contre les habitudes discontinuistes, séparatrices et de demande de penser l'oralité comme test du faire littérature, c'est-à-dire du faire voix. Ensuite, sans pour autant prétendre lancer un tournant vocal après d'autres, on se demande comment apprendre à écouter que la voix fait la vie - ce serait toute la poétique; ce qui obligerait à entendre que les vies 
demandent de faire voix. Enfin, les critiques, comme les didactiques arrimées à un tel travail poétique, éthique et politique, n'auraient qu'à accompagner au plus près les points de voix pour en augmenter les résonances: de quoi «emplir le corps de joie» et "allumer aux lèvres le sourire » pour et par des racontages, des passages de voix et d'expériences, sans fin.

This article proposes a journey in three moments. First of all, it is a question of posing the hypothesis that the voice reacts against the discontinuous, separating habits and the request to think orality like a test of the making of literature, that is to say of making the voice. Then, without pretending to launch a vocal turn after others, one wonders how to learn to listen that the voice makes life - it would be all the poetics ; which would require to hear that the lives ask to make voice. Finally, critics like didactics, stuck to such a poetic, ethical and political work, would only have to accompany the points of voice to increase the resonances : to "fill the body with joy" and "light up the smile on the lips" for and by stories, passages of voices and experiences, without end.

\section{INDEX}

Mots-clés : voix, résonance, relation, essais, poèmes, poétique, didactique de la littérature

Keywords : voice, resonance, relation, essays, poems, poetic, didactic of literature

\section{AUTEUR}

\section{SERGE MARTIN}

Université Sorbonne nouvelle Paris 3 DILTEC et THALIM

serge.martin[at]univ-paris3.fr 